\title{
The Phase of Ongoing EEG Oscillations Predicts Visual Perception
}

\author{
Niko A. Busch, ${ }^{1,2}$ Julien Dubois, ${ }^{1,2,3}$ and Rufin VanRullen ${ }^{1,2}$ \\ ${ }^{1}$ Université de Toulouse, Centre de Recherche Cerveau et Cognition, Université Paul Sabatier, France, ${ }^{2}$ Centre National de la Recherche Scientifique, \\ UMR5549, Faculté de Médecine de Rangueil, 31062 Toulouse, France, and ${ }^{3}$ California Institute of Technology, Pasadena, California 91125
}

Oscillations are ubiquitous in electrical recordings of brain activity. While the amplitude of ongoing oscillatory activity is known to correlate with various aspects of perception, the influence of oscillatory phase on perception remains unknown. In particular, since phase varies on a much faster timescale than the more sluggish amplitude fluctuations, phase effects could reveal the fine-grained neural mechanisms underlying perception. We presented brief flashes of light at the individual luminance threshold while EEG was recorded. Although the stimulus on each trial was identical, subjects detected approximately half of the flashes (hits) and entirely missed the other half (misses). Phase distributions across trials were compared between hits and misses. We found that shortly before stimulus onset, each of the two distributions exhibited significant phase concentration, but at different phase angles. This effect was strongest in the theta and alpha frequency bands. In this time-frequency range, oscillatory phase accounted for at least $16 \%$ of variability in detection performance and allowed the prediction of performance on the single-trial level. This finding indicates that the visual detection threshold fluctuates over time along with the phase of ongoing EEG activity. The results support the notion that ongoing oscillations shape our perception, possibly by providing a temporal reference frame for neural codes that rely on precise spike timing.

\section{Introduction}

Sensory systems are incessantly confronted with a continuous stream of information. Can this information be processed in an equally continuous manner at each moment in time, or does perception fluctuate between favorable and less favorable periods (VanRullen and Koch, 2003)? Ongoing oscillations may contribute to this temporal modulation of information processing, since they affect the local electrical field and the intrinsic excitability of neuronal populations (Buzsáki and Draguhn, 2004; Fries et al., 2007; Sirota et al., 2008). Indeed, tonic shifts in the power of spontaneous brain rhythms in certain frequency bands, particularly the alpha $(8-12 \mathrm{~Hz})$ and gamma $(>30 \mathrm{~Hz})$ bands, are known to accompany changes of neural response amplitude (Başar et al., 1998), attentional state (Worden et al., 2000; Sauseng et al., 2005; Thut et al., 2006; Fries et al., 2008), and perceptual performance (Ergenoglu et al., 2004; Gonzalez Andino et al., 2005; Babiloni et al., 2006; Thut et al., 2006; Womelsdorf et al., 2006; Hanslmayr et al., 2007; Romei et al., 2008; van Dijk et al., 2008). Yet oscillations are not only characterized by their power but also by their instantaneous phase. Since oscillatory phase at a given frequency reflects cyclic fluctuations of a network's excitability that occur on much shorter timescales than variations in

Received Jan. 8, 2009; revised April 2, 2009; accepted May 19, 2009.

We thank Leila Reddy and James Macdonald for their useful comments. This work was supported by grants from the Agence Nationale de la Recherche (Project ANR 06JCJC-0154), the Fyssen Foundation, and the European Young Investigator Awards to R.V. and the "European Platform for Life Sciences, Mind Sciences, and the Humanities" grant by the Volkswagen Stiftung to N.A.B.

Correspondence should be addressed to Niko A. Busch, Centre de Recherche Cerveau et Cognition, Université Paul Sabatier, 31062 Toulouse Cédex 9, France. E-mail: busch@cerco.ups-tlse.fr.

DOI:10.1523/JNEUROSCI.0113-09.2009

Copyright $\odot 2009$ Society for Neuroscience $\quad 0270-6474 / 09 / 297869-08 \$ 15.00 / 0$ oscillatory power at the same frequency (Bishop, 1932; Buzsáki and Draguhn, 2004; Lakatos et al., 2005; Klimesch et al., 2007; Lakatos et al., 2008; Montemurro et al., 2008; Rajkai et al., 2008; Sirota et al., 2008), phase effects may provide deeper insight into the fine-grained coding of sensory information. While a relationship between phase of spontaneous EEG oscillations and the amplitude of subsequent event-related potentials (ERPs) (Varela et al., 1981; Jansen and Brandt, 1991; Haig and Gordon, 1998; Makeig et al., 2002; Kruglikov and Schiff, 2003; Barry et al., 2004) or the speed of manual responses (Callaway and Yeager, 1960; Dustman and Beck, 1965; Lakatos et al., 2008) has been demonstrated, far less is known about how phase of spontaneous oscillations affects perception itself. While an influence of phase on perception could be expected based on theoretical considerations (VanRullen and Koch, 2003; Schroeder and Lakatos, 2009), EEG experiments (Monto et al., 2008) and psychophysical studies (Jones et al., 2002) have so far demonstrated these effects only for slow frequencies (i.e., frequencies $<2 \mathrm{~Hz}$ ). To understand the role of brain oscillations in perception, it is critical to assess these effects on a temporal scale that is more compatible with the temporal resolution of our visual experience (VanRullen and Koch, 2003; Holcombe, 2009). Specifically, the phase of ongoing oscillations may represent an indicator of perceptual cycles, such that a stimulus appearing at the optimal phase would be optimally registered and perceived, while at another phase it might be entirely missed.

We tested this hypothesis by investigating the influence of prestimulus oscillations of the human EEG on visual perception. We used a signal detection experiment in which visual stimuli were presented at threshold, so that on average only half of the stimuli were perceived, even though all stimuli were identical. We 
predicted that this trial-to-trial variability of perception could be systematically linked to the phase of ongoing EEG oscillations at or just preceding stimulus onset. We present a single-trial timefrequency analysis demonstrating that the phase of EEG theta $(4-8 \mathrm{~Hz})$ and alpha $(8-12 \mathrm{~Hz})$ ongoing oscillations strongly influences whether or not a stimulus is perceived.

\section{Materials and Methods}

Subjects. Fourteen participants volunteered after giving written informed consent. One participant was excluded from analysis due to excessive artifacts in the EEG data, which contaminated $>50 \%$ of the trials. Another subject was excluded due to unstable behavioral performance (hit rate out of the range of $50 \pm 25 \%$ on four of six blocks). Twelve subjects remained in the sample (four female; mean age: 27.8 years; eight right handed). All participants had normal or corrected to normal vision. The experimental protocol was approved by the Centre National de la Recherche Scientifique ethical committee.

Stimuli and procedure. Stimuli were presented on a black background on a $160 \mathrm{~Hz}$ cathode ray tube monitor. The experiment was written in Matlab using the Psychophysics Toolbox (Brainard, 1997). Subjects performed a demanding visual detection task. Each trial started with the presentation of a central fixation cross and two peripheral markers, one above and one below the position where the target stimulus was to be presented (eccentricity: $7^{\circ}$ visual angle to the right of the fixation cross). Subjects were instructed to always maintain central fixation but to pay covert attention to the site indicated by the markers. After a variable delay (range: $1000-2000 \mathrm{~ms}$ ), a target was presented on $80 \%$ of the trials (the remaining 20\% were target-absent "catch" trials introduced to estimate the false alarm rate of our participants). The target was a very small point of light (diameter: 7' visual angle) presented for $6 \mathrm{~ms}$. The target's luminance was determined for each subject before the start of the EEG session with a staircase procedure, which found the individual luminance threshold at which $50 \%$ of the stimuli were detected. After a delay of 1500 $\mathrm{ms}$ following target offset, the fixation cross turned into a question mark. Subjects were instructed to report whether or not they had perceived a target by pressing one of two buttons. No instruction for response speed was given. The button press initiated the start of the next trial. The experiment consisted of six blocks of 250 trials.

EEG acquisition and analysis. A SynAmps amplifier system (Neuroscan) was used to record EEG from 32 electrodes mounted in an elastic cap. The electrode layout was modified from the 10-20 system with an additional row of occipital electrodes and a linked-ears reference. Data were recorded in the frequency range from DC to $300 \mathrm{~Hz}$ with a sampling rate of $1000 \mathrm{~Hz}$. Electrode impedances were kept at $<5 \mathrm{k} \Omega$. Data were downsampled off-line to $500 \mathrm{~Hz}$ and epoched from $-1500 \mathrm{~ms}$ before to $1500 \mathrm{~ms}$ after stimulus onset. The horizontal electrooculogram (HEOG) was computed as the difference potential between two lateral frontal channels (F7 - F8). An automatic artifact rejection excluded epochs in which the signal exceeded $\pm 75 \mu \mathrm{V}$, and the remaining data were screened manually for residual artifacts. Event-related potentials (ERPs) were computed as the average across all trials per condition. ERPs were baseline corrected by subtracting the average of the $800 \mathrm{~ms}$ prestimulus baseline.

The analysis focused on the comparison of EEG spectral power and phases between hits and misses. Phase and power were computed by means of a continuous wavelet transform of single-trial data for the frequency range from 3 to $100 \mathrm{~Hz}$. For increased visibility, results are only plotted in the frequency range between 3 and $50 \mathrm{~Hz}$, since no significant effects were found beyond $50 \mathrm{~Hz}$. The length of the wavelets increased linearly from 3 cycles at $3 \mathrm{~Hz}$ to 8 cycles at $100 \mathrm{~Hz}$. This modified wavelet transform was selected to optimize the trade-off between temporal resolution at lower frequencies and stability at higher frequencies. At each time $t$ and frequency $f$, the result of the wavelet transform for trial $k$ is a complex number in which $A$ represents the amplitude of the signal and $\varphi$ its phase:

$$
A_{k(t, f)} e^{i \varphi_{k(t, f)}}
$$

The extent of phase concentration across trials is quantified by the intertrial coherence (ITC; also called phase-locking factor or phase-locking value).

$$
\operatorname{ITC}_{(t, f)}=\frac{1}{n} \sum_{n=1}^{k} e^{i\left(\varphi_{k(t, f)} 2 \pi\right)} .
$$

The ITC measure takes values between 0 and 1 . A value of 0 represents absence of synchronization across trials between EEG data and the timelocking events, and a value of 1 indicates perfect synchronization. ITC is computed by normalizing the lengths of the complex vectors (representing amplitude and phase) to 1 for all trials and then computing their complex average. Thus, only the information about the phase of the spectral estimate of each trial is taken into account.

We hypothesized that hits and misses were each associated with a particular phase of spontaneous EEG oscillations just before stimulus onset. Since stimulus onset is randomized and unpredictable, however, the phases of EEG oscillations can be assumed to be randomly distributed across all trials (i.e., hits and misses combined). Thus, while the distribution of phases across the whole set of trials should be random, the phase distribution of hits and misses should exhibit a stronger phase concentration. We quantified the differences in phase distributions for hits and misses by computing a phase bifurcation index $(\Phi)$. $\Phi$ is thus computed by comparing the ITC of hits and misses against the ITC of all trials:

$$
\Phi_{t, f}=\left(\mathrm{ITC}_{\mathrm{hits}(t, f)}-\mathrm{ITC}_{\mathrm{all}(t, f)}\right) \times\left(\mathrm{ITC}_{\operatorname{misses}(t, f)}-\mathrm{ITC}_{\mathrm{all}(t, f)}\right) .
$$

When phases are locked to different phase angles for hits and misses, $\Phi$ will take a positive value (Fig. 1). The upper bound of the phase bifurcation is 1 , indicating perfect phase locking in both conditions (ITC $=1$ ), but at exactly opposite phases (hence, a combined phase locking of ITC $=0$ ). The null hypothesis (random phase distributions for hits and misses) predicts a $\Phi$ close to zero. When only one condition exhibits phase locking (e.g., when ERPs are evoked only for hits after stimulus presentation), $\Phi$ takes negative values (see Fig. 1 for illustrations of these scenarios).

We computed $\Phi$ for each point in the time-frequency plane from -800 to $800 \mathrm{~ms}$ and from 3 to $100 \mathrm{~Hz}$ and statistically evaluated its magnitude with a resampling test. In a first step, data from all trials, regardless of condition, were pooled. Two sets of trials (corresponding to "hits" and "misses") were then drawn randomly from this pool, and the phase bifurcation index was computed. This procedure was repeated 500 times per subject, thus producing for each subject a distribution of $\Phi$ s based on shuffled data under the null hypothesis. In a second step, one of these pseudo- $\Phi$ s was drawn at random from the null distribution of each subject, and their grand average was computed. This procedure was repeated 100,000 times. For each point in the time-frequency plane, a $p$ value was computed as the proportion of these pseudo-grand averages that exceeded the observed grand average. This $p$ value thus indicates at which time and frequency the observed phase distributions for hits and misses are more divergent than what is expected for random data (see Fig. 3 ). The same procedure was used accordingly to evaluate the power differences between hits and misses. To correct for multiple comparisons, we analyzed the resulting distributions of $p$ values with the false discovery rate (FDR) procedure (Benjamini and Hochberg, 1995) to compute a $p$ threshold that set the expected rate of falsely rejected null hypotheses to $5 \%$.

\section{Results}

On average, subjects detected half of the targets (mean hit rate $53.7 \%$, SE 4.1), while the false alarm rate on target-absent trials (20\% of all trials) was very low (mean: $3.0 \%$, SE 0.8 ).

ERP amplitudes differed largely between perceptual conditions (see supplemental Fig. S1, available at www.jneurosci.org as supplemental material). For hits, two main ERP components were found: a negative component with a maximum at $240 \mathrm{~ms}$ at parietal electrodes contralateral to stimulation and a positive component peaking at $450 \mathrm{~ms}$ with a central topography. These 
A Two conditions phase locked at opposite phase angles; $\Phi>0$.

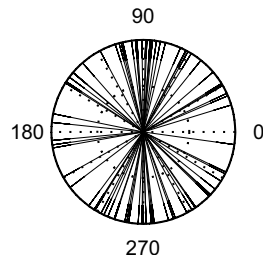

ITC all: zero

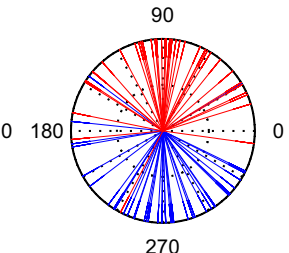

ITC1: strong ITC2: strong

\section{B Two conditions with random phases; $\Phi \approx 0$.}

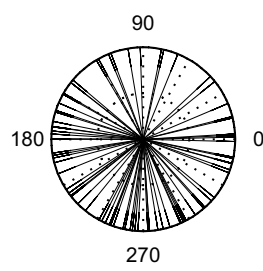

ITC all: zero

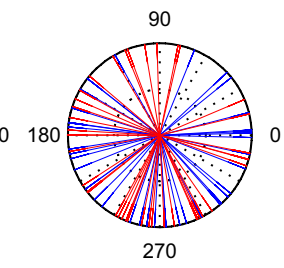

ITC1: zero ITC2: zero
$0 \quad 180$

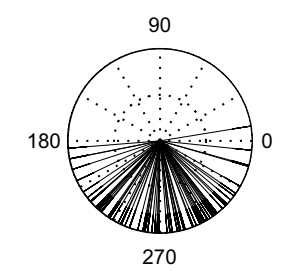

ITC all: strong one condition random; $\Phi<0$.

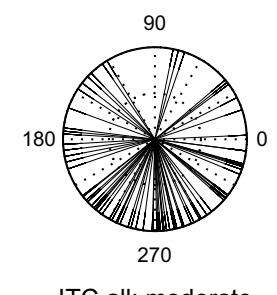

ITC all: moderate
C One condition phase locked,
270

ITC1: zero

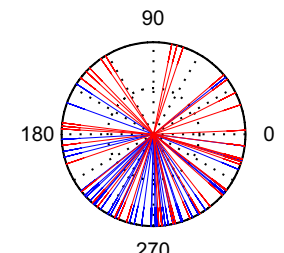

\section{Two conditions phase locked} at the same phase angle; $\Phi \approx 0$.

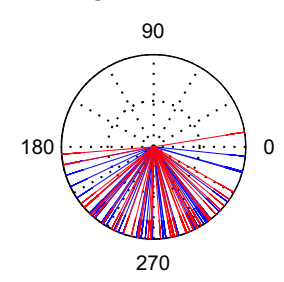

ITC1: strong ITC2: strong
Figure 1. Illustration of the phase bifurcation index with hypothetical data. The index is computed as $\Phi=\left(\mathrm{ITC}_{1}-\mathrm{ITC}_{\text {all }}\right) \times$ $\left(\right.$ ITC $_{2}-$ ITC $\left._{\text {all }}\right)$, where ITC $C_{1,2 \text { all }}$ is the intertrial coherence in conditions 1 and 2 and in both conditions combined. Each line segment represents a trial, with its angle representing phase at the frequency and time point of interest. The left circles illustrate global phase distributions, while the right circles show the two experimental conditions separately. $A$, Both conditions are phase locked at opposite angles. As a result, the ITC of both conditions combined is close to zero, resulting in a strong positive $\Phi$. This is equivalent to the hypothesis that hits and misses are each associated with different phase angles. $\boldsymbol{B}$, The null hypothesis states that both conditions are randomly distributed, resulting in a $\Phi$ close to zero. C, Only one condition is phase locked, and the other is randomly distributed. The resulting ITC of both conditions is stronger than the ITC of condition 1, but smaller than the ITC of condition 2, resulting in a negative $\Phi$. This situation is expected during the ERP time range, where an ERP is present only for detected stimuli. $\boldsymbol{D}$, Both conditions are strongly phase locked at the same phase angle; the resulting $\Phi$ is close to zero.

ing missed targets than detected targets (Fig. 3A), and this effect reached a maximum at $8.2 \mathrm{~Hz}$ and $-492 \mathrm{~ms}$ preceding stimulus onset. To statistically confirm these effects, we used a resampling test and applied the FDR method (Benjamini and Hochberg, 1995) to correct for the effect of multiple comparisons across time points and frequencies. Effects that satisfied a 5\% FDR criterion are outlined in white in Figure $3 A$. The impact of spectral power on detection performance was assessed by binning single trials according to the spectral power at that time-frequency point and computing the average detection rate of our observers in each of 10 bins. To minimize the impact of individual variations in performance, detection rate was standardized for each observer by dividing the hit rate in each bin by the average hit rate of that observer. Standardized hit rates were highest in the bin corresponding to lowest spectral power, and lowest in the bin with the highest power. The difference between these two bins amounted to a $12 \%$ change in performance, and was confirmed statistically with a repeated measurements ANOVA with "bin" as a factor $(10$ levels $): F_{(9,99)}=2.404 ; p=$ 0.0165 .

A similar analysis was performed for the phase bifurcation index $\Phi$-a measure of the divergence of two phase distributions (Fig. 1). The phase bifurcation spectrum (averaged across channels and time

ERPs were virtually absent when the target was not perceived. The differences were confirmed by a paired $t$ test computed on the mean amplitude from 230 to $250 \mathrm{~ms}$ at channel T5 $\left(t_{(11)}=\right.$ $-3.07 ; p=0.01)$ and on the mean amplitude from 430 to $470 \mathrm{~ms}$ at channel $\mathrm{Cz}\left(t_{(11)}=9.71 ; p<0.001\right)$.

We investigated whether ongoing EEG activity before stimulus presentation influences detection by analyzing spectral power and phase in the prestimulus time window. In particular, we (1) analyzed power differences between hit and miss trials and (2) computed the phase bifurcation index (see Materials and Methods and Fig. 1); significantly positive phase bifurcation indicates that oscillations are locked to different phase angles on hit and miss trials. While a power difference in the prestimulus period is expected based on previous studies, an effect of prestimulus phase on subsequent perception would constitute a major conceptual advance.

We started out by computing spectra of power effects and phase bifurcation as averages across electrodes and time points in the $800 \mathrm{~ms}$ prestimulus interval (Fig. 2). These pooled measures allowed us to determine frequency bands of interest, on which more fine-grained analyses were then performed. We found that spectral power was significantly stronger for misses than for hits in the $6-12 \mathrm{~Hz}$ frequency range (Fig. $2 \mathrm{~A}$, left inset). In this frequency range, the effect was strongest in the -600 to $-300 \mathrm{~ms}$ time window preceding stimulus onset (Fig. $2 \mathrm{~A}$, bottom inset). In this time-frequency window, a widely distributed topography was observed with a maximum at the frontocentral channel Fz. Subsequent power analyses were thus focused on this channel. We found that spectral power was significantly stronger preced- points in the prestimulus interval) was strongest in a frequency range from 6 to $10 \mathrm{~Hz}$. Here, effects exceeded the $95 \%$ confidence limit that was based on 100,000 synthetic datasets with random phase distributions (Fig. $2 \mathrm{~B}$, left inset). Strongest phase bifurcation was observed in the -300 to $-50 \mathrm{~ms}$ time window preceding stimulus onset (Fig. 2B, bottom inset), indicating significant phase concentration for hits and misses, but at different phase angles. We ascertained that phases were uniformly distributed across all trials (i.e., when hits and misses were combined) for this time-frequency point with a Rayleigh test (cf. Fisher, 1995). When $p$ values were Bonferroni-corrected for multiple comparisons, none of the subjects had a significant deviation from uniformity, but without correction for multiple comparisons the deviation was statistically significant for 1 out of the 12 subjects. To assess the significance of this finding, we performed a randomization test by computing a distribution of random phases for each subject with the same number of "trials" as in the actual data, and the Rayleigh test was computed for these random phases. This procedure was repeated 100,000 times for the group of 12 subjects. The probability of observing at least 1 out of 12 significant deviations from uniformity was 0.46 , even though phases were now drawn from a random distribution. Thus, observing only one significant deviation from uniformity in our dataset is highly expected even for randomly distributed phases. We thus conclude that it is safe to assume that phases were indeed uniformly distributed in our data.

The phase bifurcation effect in this time-frequency range had a frontocentral topography with a maximum at channel Fz. We 
evaluated the statistical significance of this effect at channel $\mathrm{Fz}$ with a resampling test and found the strongest effect at $7.1 \mathrm{~Hz}$ and $120 \mathrm{~ms}$ preceding stimulus onset (Fig. $3 B)$. We used the FDR method to correct for multiple comparisons; white outlines in the inset of Figure $3 B$ indicate the 55 time-frequency points that passed the $5 \%$ FDR criterion (meaning that only 3 points out of these 55 are expected to be false positives due to multiple comparisons). To verify that these results were not due to a single outlier in our subject pool, significance maps were recomputed 12 times, each time leaving out a different subject; the region of maximum significance (using an FDR of 0.1) always counted at least nine time-frequency points, every time including the point of interest at $7.1 \mathrm{~Hz}$ and $-120 \mathrm{~ms}$ preceding stimulus onset.

Phases at this time-frequency point were pooled into 11 bins. The effect of phase bifurcation was significant across subjects, and the mean phase values for hits and for misses were approximately similar across subjects (Fig. 3B, top inset). However, the exact phase at which performance was highest could vary slightly between subjects. We thus adjusted each subject's phase distributions such that for each subject the phase at which performance was best was aligned to a phase angle of zero. Thus, a trivial feature of the resulting distribution is a peak exceeding the standardized average hit rate at a phase of zero. However, we also found that performance decreased monotonically to a minimum at the opposite phase-a nontrivial property confirming our hypothesis that hits and misses are associated with opposite phase angles. Comparing the average of the two bins adjacent to the zero bin with the average of the two bins at the opposite phase revealed that phase accounted for a difference of $16 \%$ of performance (standardized performance: 1.05 vs 0.89 ), i.e., even stronger than the corresponding effect observed for power (Fig. 3A). This effect was confirmed by an ANOVA with a 10-level "bin" factor (leaving out the bin centered on zero): $F_{(9,99)}=$ 2.696; $p=0.0076$.

The influence of EEG phase on performance was independently assessed using a linear classifier approach: a support vector machine classifier was trained to discriminate hits versus miss trials based on instantaneous phase at electrode Fz. Classifier output on a distinct set of test trials was maximally correlated with observers' performance in the time-frequency window around $7 \mathrm{~Hz},-120 \mathrm{~ms}$ (supplemental Fig. S2, available at www. jneurosci.org as supplemental material).

These analyses relied on a time-frequency transform that convolves the signal with a wavelet function, which is extended in time and the spread of which is inversely related to the frequency being analyzed. Thus, it is conceivable that some of the reported
Power (hits vs. misses)

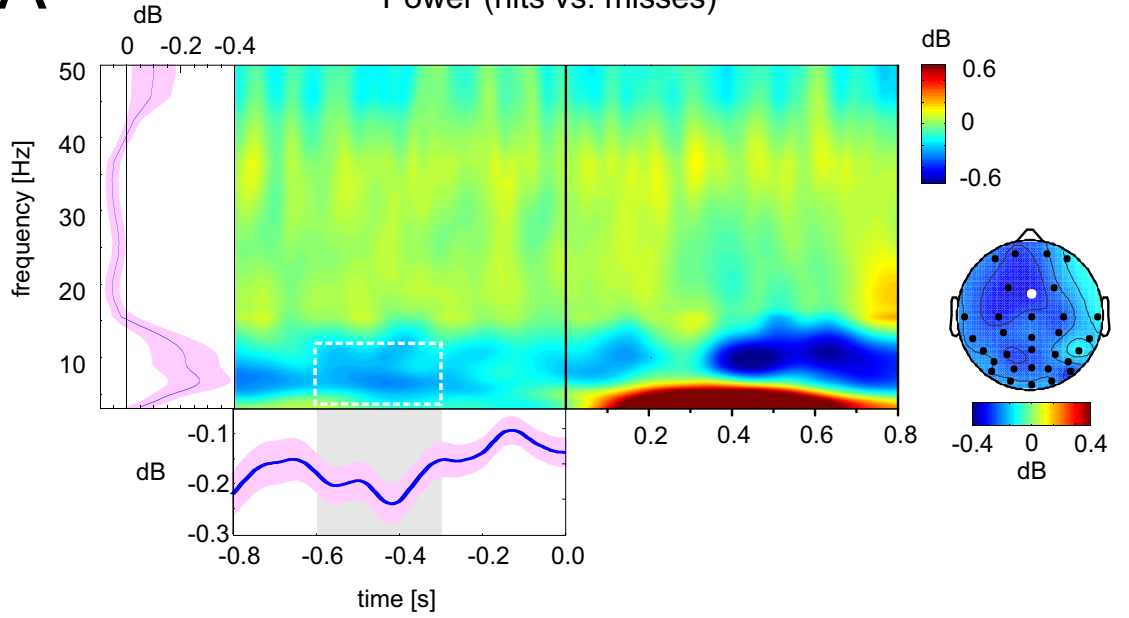

Phase bifurcation index

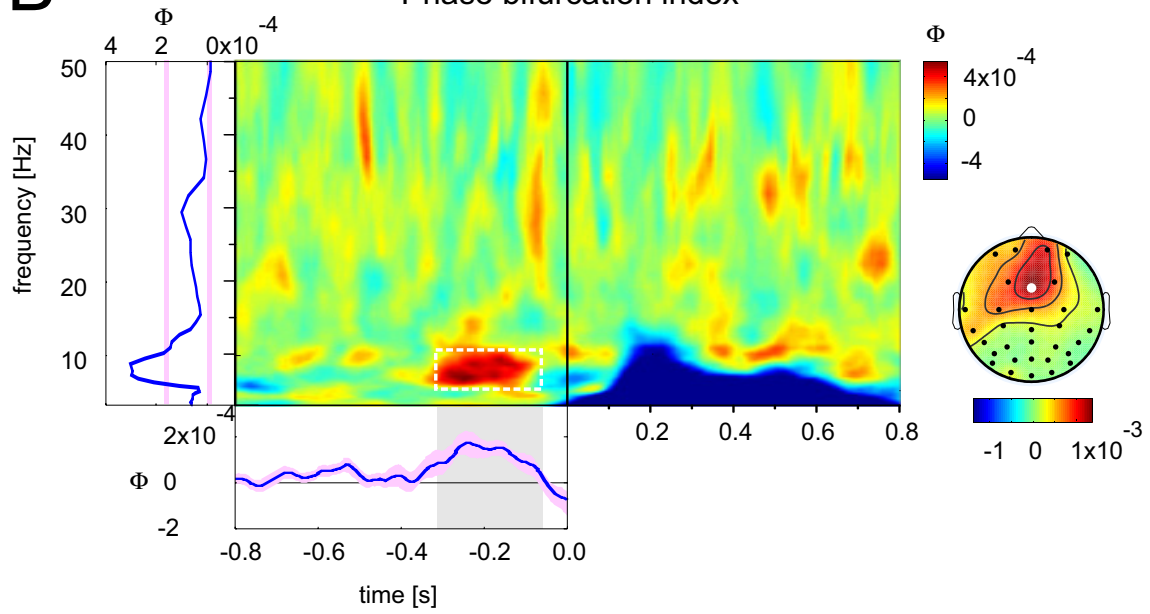

Figure 2. Raw effects of oscillatory power and phase. A, Difference in spectral power in decibels between hits and misses, averaged across channels and subjects. Negative values indicate stronger power for misses. Left inset shows power difference averaged across time points in the prestimulus window (shaded areas: SEM). The main difference is found in the $6-12 \mathrm{~Hz}$ frequency range. Bottom inset, Power difference averaged across frequencies in this range, with a maximally negative difference power difference from 6 to $12 \mathrm{~Hz}$ and from -600 to $-300 \mathrm{~ms}$ preceding stimulus onset. $\boldsymbol{B}$, Phase bifurcation index $(\Phi)$, averaged cross all channels and subjects. Positive values indicate that phase distributions are locked to different phase angles for hits and cking exclusively for hits in the ERP time range). Left inset shows $\Phi$ averaged across all time points in the prestimulus window area: SE). Phase bifurcation is strongest from -300 to $-50 \mathrm{~ms}$ preceding stimulus onset (gray shaded area). The topography shows the distribution of $\Phi$ from 6 to $10 \mathrm{~Hz}$ and from -300 to -50 ms preceding stimulus onset.

prestimulus effects were affected by EEG data collected after stimulus onset. However, we repeated the analysis with much shorter wavelet functions (limited to 1 cycle instead of 3 or more cycles) and confirmed that the prestimulus effects of phase and power precede the time range that can potentially be affected by poststimulus data (compare supplemental Fig. S3, available at www.jneurosci.org as supplemental material).

We also ascertained that power and phase effects were not due to any eye movement artifacts before stimulus onset that survived the artifact rejection procedure. For example, differences in power and phase on hit and miss trials could have simply been caused by differences in the number or the timing of eye blinks before stimulus onset. Eye blinks are associated with high amplitude signals at frontal channels. However, we found no evidence 

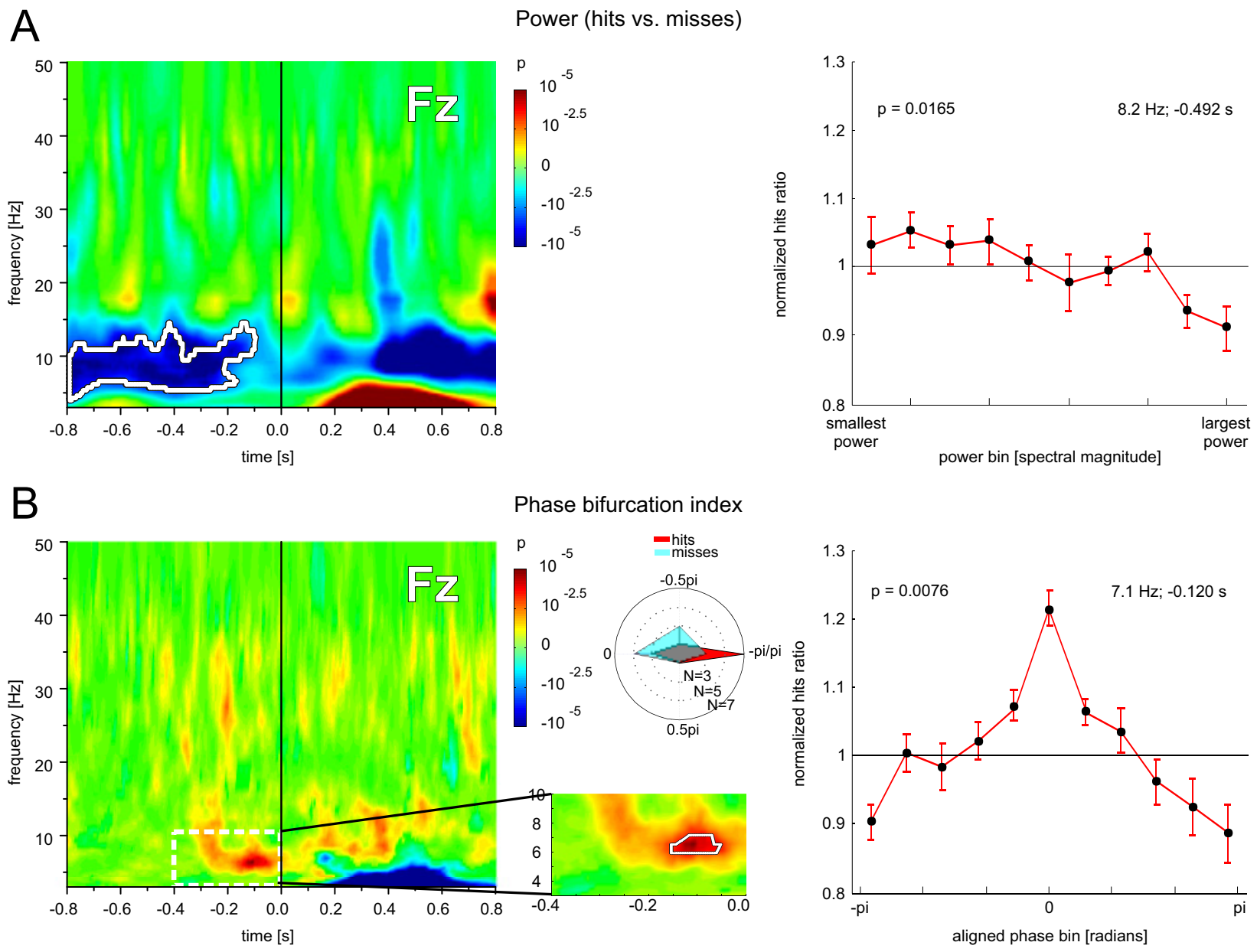

Figure 3. Relation between perception and oscillatory activity. $\boldsymbol{A}$, Statistical significance of the power difference at channel Fz between hits and misses (left panel), evaluated with a resampling test. Alpha power $(6-12 \mathrm{~Hz})$ preceding stimulus onset was significantly stronger for misses than for hits. The color map represents uncorrected $p$ values and the white outline delimits significant effects corresponding to an FDR of $5 \%$. The right panel shows the relationship between spectral power in the alpha band $(8.2 \mathrm{~Hz})$ at $-492 \mathrm{~ms}$ and performance after single trials were pooled in 10 power bins. The horizontal line indicates average performance (standardized to 1) across all bins. Error bars represent SEM. Performance is superior on trials with lowest alpha power (1-way ANOVA, $p<0.0165$ ). $\boldsymbol{B}$, Statistical significance of phase bifurcation between hits and misses at channel $\mathrm{Fz}$ (left panel); axes and conventions are as in $\boldsymbol{A}$. At $\sim 7 \mathrm{~Hz}$ and $-120 \mathrm{~ms}$ prestimulus, hits and misses are associated with different phase angles. Top inset, The circular histograms of mean phase angles at $7.1 \mathrm{~Hz}$ and $120 \mathrm{~ms}$ for hits and misses across participants. Phases were pooled into four phase bins. The distance from the origin indicates the number of subjects falling within a bin, and the angles indicate the lower bound of each phase bin. Bottom inset, Close-up on the 55 time-frequency points with significant phase bifurcation that satisfy an FDR of 5\%. The right panel shows the relationship between phase (at $7.1 \mathrm{~Hz} ;-120 \mathrm{~ms}$ ) and standardized performance after phases were aligned for each subject so that the optimal phase corresponds to a zero phase angle. Performance declines to a minimum at the opposite phase angle (1-way ANOVA, $p<0.01$ ).

for eye blinks on single trials or in the average ERP, either at Fz (where power and phase bifurcation effects were strongest) or in the HEOG (compare supplemental Fig. S4, available at www. jneurosci.org as supplemental material), for hits or misses. Alternatively, hits and misses might have been associated with horizontal saccades toward or away from the target, respectively. If so, these saccades might have introduced an electrical artifact with opposite polarity (i.e., opposite phase angles) for hits and misses. However, inspection of the HEOG on single trials and in the averaged ERP did not reveal any signs of systematic prestimulus saccades, least of all in opposite directions (compare supplemental Fig. S4, available at www.jneurosci.org as supplemental material). In sum, these results indicate that power and phase bifurcation effects in the main analysis were not brought about by ocular artifacts.

\section{Discussion}

The traditional approach in cognitive neuroscience has been to investigate neural activity in response to (that is, succeeding) an experimental event. With this reasoning, it is usually assumed that the brain state preceding the event does not play a meaning- ful role in how the event will be processed, and that trial-to-trial variations in the response to identical stimuli reflect random noise. In recent years however, there has been a growing interest in the role of prestimulus brain states in many different domains of research. Examples include prestimulus shifts of firing rates and synchronization (Fries et al., 2001; Womelsdorf et al., 2006), anticipatory baseline changes of blood oxygenation leveldependent activity in functional magnetic resonance imaging (McMains et al., 2007; Silver et al., 2007; Sestieri et al., 2008), spatiotemporal activity patterns (Arieli et al., 1996), and spontaneous EEG activity (Başar et al., 1998; Ergenoglu et al., 2004; Lakatos et al., 2005; Thut et al., 2006; Hanslmayr et al., 2007; Monto et al., 2008; van Dijk et al., 2008; Schroeder and Lakatos, 2009). We investigated the influence of prestimulus oscillations on visual perception. We presented visual stimuli near threshold, and found that their detection probability was strongly dependent on the phase of spontaneous EEG oscillations in the low alpha and theta bands just before stimulus onset. This result suggests that perception may operate in successive periodic cycles, 
alternating between phases of optimal excitability where threshold stimuli are consciously perceived, and phases associated with stronger inhibition at which the same stimuli are more likely to escape detection.

Our analysis also confirmed previous studies reporting that stimuli preceded by strong alpha power are less likely to be detected (Ergenoglu et al., 2004; Babiloni et al., 2006; Thut et al., 2006; Hanslmayr et al., 2007). However, the influence of phase on visual detection performance, accounting for a minimum of $16 \%$ variability in performance (Fig. $3 B$ ), was in fact stronger than the previously characterized effect of alpha power, which in our study accounted for $12 \%$ of performance variability. While these numbers may appear small, it is important to remember that our estimates rely on single-trial EEG data, which in general are deemed to be inherently too noisy to be analyzed (Picton et al., 2000). The measured effects of phase and power were largely independent, as indicated by the different topographies and time courses: while alpha power effects prevailed throughout the prestimulus time window and were strongest $\sim 500 \mathrm{~ms}$ before stimulus onset, the effect of phase on perception was restricted to the interval immediately preceding stimulus onset. In line with this distinction, it should be noted that the effects of prestimulus power (Ergenoglu et al., 2004) or interelectrode synchrony (Hanslmayr et al., 2007) on performance are related to the subject's tonic state of attention or arousal, which usually fluctuates on a much longer timescale than the rhythmic modulations of excitability associated with oscillatory phase (Makeig and Jung, 1996; Klimesch et al., 2007). Thus, while spontaneous power of neural oscillations undoubtedly influences visual perception, the effects of prestimulus phase may be more closely linked to the actual coding and processing of visual information.

The existence of a relationship between spontaneous EEG alpha phase and the amplitude of subsequent ERPs (Varela et al., 1981; Jansen and Brandt, 1991; Haig and Gordon, 1998; Makeig et al., 2002; Kruglikov and Schiff, 2003; Barry et al., 2004) or the speed of manual responses (Callaway and Yeager, 1960; Dustman and Beck, 1965) has been postulated for decades. However, this is rarely taken into account in theories of visual perception, perhaps because it could be attributed to a mere facilitation at the biophysical level, without direct relevance to conscious perception. In contrast, our study directly demonstrates that prestimulus phase of ongoing neural oscillations can affect visual perception itself. Note that in our study the major effect was found at $\sim 7 \mathrm{~Hz}$, i.e., at the intersection between theta and alpha frequency bands, whereas most of the previous studies mentioned above reported their effects in the alpha band-the frequency range from 8 to 12 $\mathrm{Hz}$. This apparent divergence may be explained by the wide range of frequencies that were analyzed without restriction in our study, while previous studies tended to limit their analysis to a narrow frequency range by application of a preselected bandpass filter (usually centered around $10 \mathrm{~Hz}$ ) (Dustman and Beck, 1965; Varela et al., 1981; Jansen and Brandt, 1991; Haig and Gordon, 1998). In addition, the absence of significant phase effects in the gamma band $(>30 \mathrm{~Hz})$ is worth mentioning, since such effects might have been expected on theoretical grounds (Fries et al., 2007). However, this absence may not rule out a role for gamma oscillations, but could instead reflect an intrinsic limitation of surface EEG recordings: small shifts of conduction delays, synaptic transmission delays, and other biophysical parameters on the order of a few milliseconds exert a maximally disruptive influence on the phase measured at higher frequencies. For example, a mere $8 \mathrm{~ms}$ shift actually corresponds to a full phase reversal for an oscillation at $60 \mathrm{~Hz}$. Moreover, the signal-to-noise ratio in sur- face EEG recordings decreases at higher frequencies. In addition, amplitude at high frequencies is often coupled to the phase of lower frequencies (Lakatos et al., 2005), making gamma activity detectable only in certain time windows. Therefore, the gamma phase recorded at the scalp surface may not always faithfully reflect the relevant neuronal oscillation. Similarly, we could not assess effects of slow frequencies (i.e., slower than $3 \mathrm{~Hz}$ ), which would have required much longer intertrial intervals. It has been demonstrated recently that detection of somatosensory stimuli is strongly correlated with the phase of frequencies below $1 \mathrm{~Hz}$ (Monto et al., 2008), and future studies should address this phenomenon in the visual domain. It may be speculated that such effects are strongest in experiments with inherent temporal structure and predictable stimulus onsets, which would allow a sensory system to use a low frequency oscillation to prepare the excitability state of the system for the approximate time of stimulus arrival [for similar ideas, see Jones et al. (2002), Lakatos et al. (2008), and Schroeder and Lakatos (2009)].

What could be the source of the effect of EEG phase on visual perception reported here? Given its frontocentral topography and its frequency range in the theta and low alpha band, one might conjecture a source within frontal midline structures. These areas have previously been discussed as generators of EEG theta rhythms (Debener et al., 2005; Onton et al., 2005; Tsujimoto et al., 2006), which are involved in a variety of cognitive tasks (for review, see Mitchell et al., 2008) requiring sustained attention (Sauseng et al., 2007) or working memory (Gevins et al., 1997; Jensen and Tesche, 2002), and are correlated with manual reaction times (Gonzalez Andino et al., 2005). Therefore, it may not be surprising that frontal theta rhythms could also participate in the dynamic shaping of perceptual experience. However, it should be emphasized that the topography in Figure $2 B$ corresponds to a map of the effects' statistical significance, without providing information about the polarity of the underlying electrical signals. Such polarity information is essential for localizing neuroelectric sources. Moreover, the present data were recorded from only 32 channels, while source localization requires a substantially larger number of channels. Thus, any conjecture as to the cortical generators of the phase effects has to remain speculative at present. Future studies should apply similar phase analyses in source space using denser electrode montages.

To conclude, the finding that threshold stimuli are sometimes perceived and sometimes missed depending on the phase of spontaneous brain oscillations supports the assumption that these oscillations reflect cyclic variations of neural excitability (Bishop, 1932; Harter, 1967; Buzsáki and Draguhn, 2004; VanRullen et al., 2005; Fries et al., 2007; Rajkai et al., 2008; Sirota et al., 2008). Such excitability cycles may instantiate a discrete processing mode, framing visual perception into discrete epochs or "perceptual moments" (Stroud, 1967; Varela et al., 1981; Purves et al., 1996; VanRullen and Koch, 2003; Smith et al., 2006). The advantage of a discrete processing scheme could be to transform stimulus information into a temporal code. Indeed, in a network whose excitability is modulated by spontaneous oscillations, the relative strength of input stimulus features is converted into a phase code: the stronger the feature value, the earlier the corresponding units will respond within a given oscillation cycle (O'Keefe and Recce, 1993; Hopfield, 1995; Mehta et al., 2002; Margrie and Schaefer, 2003; Buzsáki and Draguhn, 2004; Lisman, 2005; Fries et al., 2007). The hippocampus heavily relies on this principle (coined "theta phase precession") to encode spatial location as the animal navigates through the spatially restricted firing field of so-called "place cells" (O'Keefe and Recce, 1993; 
Dragoi and Buzsáki, 2006). Similarly, visual attributes of a stimulus could be represented in each oscillation cycle by the relative phases of firing across the neuronal population (Thorpe et al., 2001; VanRullen et al., 2005; Fries et al., 2007). While further electrophysiological evidence will be needed to support this speculative proposal, the present demonstration of periodic fluctuations of visual perceptual abilities could constitute an important first step in this direction.

\section{References}

Arieli A, Sterkin A, Grinvald A, Aertsen A (1996) Dynamics of ongoing activity: explanation of the large variability in evoked cortical responses. Science 273:1868-1871.

Babiloni C, Brancucci A, Del Percio C, Capotosto P, Arendt-Nielsen L, Chen ACN, Rossini PM (2006) Anticipatory electroencephalography alpha rhythm predicts subjective perception of pain intensity. J Pain 7:709-717.

Barry RJ, Rushby JA, Johnstone SJ, Clarke AR, Croft RJ, Lawrence CA (2004) Eventrelated potentials in the auditory oddball as a function of EEG alpha phase at stimulus onset. Clin Neurophysiol 115:2593-2601.

Başar E, Rahn E, Demiralp T, Schürmann M (1998) Spontaneous EEG theta activity controls frontal visual evoked potential amplitudes. Electroencephalogr Clin Neurophysiol 108:101-109.

Benjamini Y, Hochberg Y (1995) Controlling the false discovery rate: a practical and powerful approach to multiple testing. J R Stat Soc Series B 57:289-300.

Bishop GH (1932) Cyclic changes in excitability of the optic pathway of the rabbit. Am J Physiol 103:213-224.

Brainard DH (1997) The psychophysics toolbox. Spat Vis 10:433-436.

Buzsáki G, Draguhn A (2004) Neuronal oscillations in cortical networks. Science 304:1926-1929.

Callaway E 3rd, Yeager CL (1960) Relationship between reaction time and electroencephalographic alpha phase. Science 132:1765-1766.

Debener S, Ullsperger M, Siegel M, Fiehler K, von Cramon DY, Engel AK (2005) Trial-by-trial coupling of concurrent electroencephalogram and functional magnetic resonance imaging identifies the dynamics of performance monitoring. J Neurosci 25:11730-11737.

Dragoi G, Buzsáki G (2006) Temporal encoding of place sequences by hippocampal cell assemblies. Neuron 50:145-157.

Dustman RE, Beck EC (1965) Phase of alpha brain waves, reaction time and visually evoked potentials. Electroencephalogr Clin Neurophysiol 18:433-440.

Ergenoglu T, Demiralp T, Bayraktaroglu Z, Ergen M, Beydagi H, Uresin Y (2004) Alpha rhythm of the EEG modulates visual detection performance in humans. Brain Res Cogn Brain Res 20:376-383.

Fisher NI (1995) Statistical analysis of circular data. Cambridge, UK: Cambridge UP.

Fries P, Neuenschwander S, Engel AK, Goebel R, Singer W (2001) Rapid feature selective neuronal synchronization through correlated latency shifting. Nat Neurosci 4:194-200.

Fries P, Nikolić D, Singer W (2007) The gamma cycle. Trends Neurosci 30:309-316.

Fries P, Womelsdorf T, Oostenveld R, Desimone R (2008) The effects of visual stimulation and selective visual attention on rhythmic neuronal synchronization in macaque area V4. J Neurosci 28:4823-4835.

Gevins A, Smith ME, McEvoy L, Yu D (1997) High-resolution EEG mapping of cortical activation related to working memory: effects of task difficulty, type of processing, and practice. Cereb Cortex 7:374-385.

Gonzalez Andino SL, Michel CM, Thut G, Landis T, Grave de Peralta R (2005) Prediction of response speed by anticipatory high-frequency (gamma band) oscillations in the human brain. Hum Brain Mapp 24:50-58.

Haig AR, Gordon E (1998) EEG alpha phase at stimulus onset significantly affects the amplitude of the P3 ERP component. Int J Neurosci 93:101-115

Hanslmayr S, Aslan A, Staudigl T, Klimesch W, Herrmann CS, Bäuml KH (2007) Prestimulus oscillations predict visual perception performance between and within subjects. Neuroimage 37:1465-1473.

Harter MR (1967) Excitability cycles and cortical scanning: a review of two hypotheses of central intermittency in perception. Psychol Bull 68:47-58.

Holcombe AO (2009) Seeing slow and seeing fast: two limits on perception. Trends Cogn Sci 13:216-221.
Hopfield JJ (1995) Pattern recognition computation using action potential timing for stimulus representation. Nature 376:33-36.

Jansen BH, Brandt ME (1991) The effect of the phase of prestimulus alpha activity on the averaged visual evoked response. Electroencephalogr Clin Neurophysiol 80:241-250.

Jensen O, Tesche CD (2002) Frontal theta activity in humans increases with memory load in a working memory task. Eur J Neurosci 15:1395-1399.

Jones MR, Moynihan H, MacKenzie N, Puente J (2002) Temporal aspects of stimulus-driven attending in dynamic arrays. Psychol Sci 13:313-319.

Klimesch W, Sauseng P, Hanslmayr S (2007) EEG alpha oscillations: the inhibition-timing hypothesis. Brain Res Rev 53:63-88.

Kruglikov SY, Schiff SJ (2003) Interplay of electroencephalogram phase and auditory-evoked neural activity. J Neurosci 23:10122-10127.

Lakatos P, Shah AS, Knuth KH, Ulbert I, Karmos G, Schroeder CE (2005) An oscillatory hierarchy controlling neuronal excitability and stimulus processing in the auditory cortex. J Neurophysiol 94:1904-1911.

Lakatos P, Karmos G, Mehta AD, Ulbert I, Schroeder CE (2008) Entrainment of neuronal oscillations as a mechanism of attentional selection. Science 320:110-113.

Lisman J (2005) The theta/gamma discrete phase code occuring during the hippocampal phase precession may be a more general brain coding scheme. Hippocampus 15:913-922.

Makeig S, Jung TP (1996) Tonic, phasic, and transient EEG correlates of auditory awareness in drowsiness. Brain Res Cogn Brain Res 4:15-25.

Makeig S, Westerfield M, Jung TP, Enghoff S, Townsend J, Courchesne E, Sejnowski TJ (2002) Dynamic brain sources of visual evoked responses. Science 295:690-694.

Margrie TW, Schaefer AT (2003) Theta oscillation coupled spike latencies yield computational vigour in a mammalian sensory system. J Physiol 546:363-374.

McMains SA, Fehd HM, Emmanouil TA, Kastner S (2007) Mechanisms of feature- and space-based attention: response modulation and baseline increases. J Neurophysiol 98:2110-2121.

Mehta MR, Lee AK, Wilson MA (2002) Role of experience and oscillations in transforming a rate code into a temporal code. Nature 417:741-746.

Mitchell DJ, McNaughton N, Flanagan D, Kirk IJ (2008) A review of frontalmidline theta from the perspective of hippocampal "theta". Prog Neurobiol 86:156-185.

Montemurro MA, Rasch MJ, Murayama Y, Logothetis NK, Panzeri S (2008) Phase-of-firing coding of natural visual stimuli in primary visual cortex. Curr Biol 18:375-380.

Monto S, Palva S, Voipio J, Palva JM (2008) Very slow EEG fluctuations predict the dynamics of stimulus detection and oscillation amplitudes in humans. J Neurosci 28:8268-8272.

O’Keefe J, Recce ML (1993) Phase relationship between hippocampal place units and the EEG theta rhythm. Hippocampus 3:317-330.

Onton J, Delorme A, Makeig S (2005) Frontal midline EEG dynamics during working memory. Neuroimage 27:341-356.

Picton TW, Bentin S, Berg P, Donchin E, Hillyard SA, Johnson R Jr, Miller GA, Ritter W, Ruchkin DS, Rugg MD, Taylor MJ (2000) Guidelines for using human event-related potentials to study cognition: recording standards and publication criteria. Psychophysiology 37:127-152.

Purves D, Paydarfar JA, Andrews TJ (1996) The wagon wheel illusion in movies and reality. Proc Natl Acad Sci U S A 93:3693-3697.

Rajkai C, Lakatos P, Chen CM, Pincze Z, Karmos G, Schroeder CE (2008) Transient cortical excitation at the onset of visual fixation. Cereb Cortex 18:200-209.

Romei V, Brodbeck V, Michel C, Amedi A, Pascual-Leone A, Thut G (2008) Spontaneous fluctuations in posterior alpha-band EEG activity reflect variability in excitability of human visual areas. Cereb Cortex 18:2010-2018.

Sauseng P, Klimesch W, Stadler W, Schabus M, Doppelmayr M, Hanslmayr S, Gruber WR, Birbaumer N (2005) A shift of visual spatial attention is selectively associated with human EEG alpha activity. Eur J Neurosci 22:2917-2926.

Sauseng P, Hoppe J, Klimesch W, Gerloff C, Hummel FC (2007) Dissociation of sustained attention from central executive functions: local activity and interregional connectivity in the theta range. Eur J Neurosci 25:587-593.

Schroeder CE, Lakatos P (2009) Low-frequency neuronal oscillations as instruments of sensory selection. Trends Neurosci 32:9-18.

Sestieri C, Sylvester CM, Jack AI, d'Avossa G, Shulman GL, Corbetta M 
(2008) Independence of anticipatory signals for spatial attention from number of nontarget stimuli in the visual field. J Neurophysiol 100:829-838.

Silver MA, Ress D, Heeger DJ (2007) Neural correlates of sustained spatial attention in human early visual cortex. J Neurophysiol 97:229-237.

Sirota A, Montgomery S, Fujisawa S, Isomura Y, Zugaro M, Buzsáki G (2008) Entrainment of neocortical neurons and gamma oscillations by the hippocampal theta rhythm. Neuron 60:683-697.

Smith ML, Gosselin F, Schyns PG (2006) Perceptual moments of conscious visual experience inferred from oscillatory brain activity. Proc Natl Acad Sci U S A 103:5626-5631.

Stroud JN (1967) The fine structure of psychological time. Ann N Y Acad Sci 138:623-631.

Thorpe S, Delorme A, Van Rullen R (2001) Spike-based strategies for rapid processing. Neural Netw 14:715-725.

Thut G, Nietzel A, Brandt SA, Pascual-Leone A (2006) Alpha-band electroencephalographic activity over occipital cortex indexes visuospatial attention bias and predicts visual target detection. J Neurosci 26:9494-9502.

Tsujimoto T, Shimazu H, Isomura Y (2006) Direct recording of theta oscil- lations in primate prefrontal and anterior cingulate cortices. J Neurophysiol 95:2987-3000.

van Dijk H, Schoffelen JM, Oostenveld R, Jensen O (2008) Prestimulus oscillatory activity in the alpha band predicts visual discrimination ability. J Neurosci 28:1816-1823.

VanRullen R, Koch C (2003) Is perception discrete or continuous? Trends Cogn Sci 7:207-213.

VanRullen R, Guyonneau R, Thorpe SJ (2005) Spike times make sense. Trends Neurosci 28:1-4.

Varela FJ, Toro A, John ER, Schwartz EL (1981) Perceptual framing and cortical alpha rhythm. Neuropsychologia 19:675-686.

Womelsdorf T, Fries P, Mitra PP, Desimone R (2006) Gamma-band synchronization in visual cortex predicts speed of change detection. Nature 439:733-736.

Worden MS, Foxe JJ, Wang N, Simpson GV (2000) Anticipatory biasing of visuospatial attention indexed by retinotopically specific alphaband electroencephalography increases over occipital cortex. J Neurosci 20:RC63. 